\title{
Cortical Thickness and Gyrification Index in Eating Disorders
}

\author{
Cascino G. ${ }^{1}$, Canna A. ${ }^{2}$, Russo A.G. ${ }^{2}$, Monteleone A.M. ${ }^{1}$, Prinster A. ${ }^{3}$, Esposito F. $^{2}$, Di Salle F. ${ }^{2}$, Monteleone P. ${ }^{2}$ \\ ${ }^{I}$ Department of Psychiatry, University of Campania "Luigi Vanvitelli", Naples, Italy \\ ${ }^{2}$ Department of Medicine, Surgery and Dentistry "Scuola Medica Salernitana", University of Salerno, Salerno, Italy \\ ${ }^{3}$ IRCCS SDN Research Institute, Department of Radiology and Nuclear Medicine, Naples, Italy.
}

\section{AIM OF THE STUDY}

Eating disorders (EDs) are psychiatric diseases with possible neurodevelopmental etiopathogenesis [1;2]. Aim of this study was to estimate and compare brain regional cortical thickness (CT) and gyrification index (GI), specific markers of deviations from normal neurodevelopment, in females with EDs and healthy controls.

\section{METHODS}

Twenty-two underweight anorexia nervosa (AN), 10 weight recovered anorexia nervosa (recAN), 24 bulimia nervosa (BN) women and 35 healthy controls (HC) underwent a 3T MRI scan. All data were processed by FreeSurfer.

\section{RESULTS}

Significant differences were found in CT of left and right lateral orbitofrontal cortex (OFC) and right medial OFC between AN and recAN groups. AN group showed reduced CT values in left inferior temporal regions compared to HC. Significant positive correlations were found between body mass index (BMI) and CT in left and right lateral OFC and right medial OFC in AN and recAN groups.

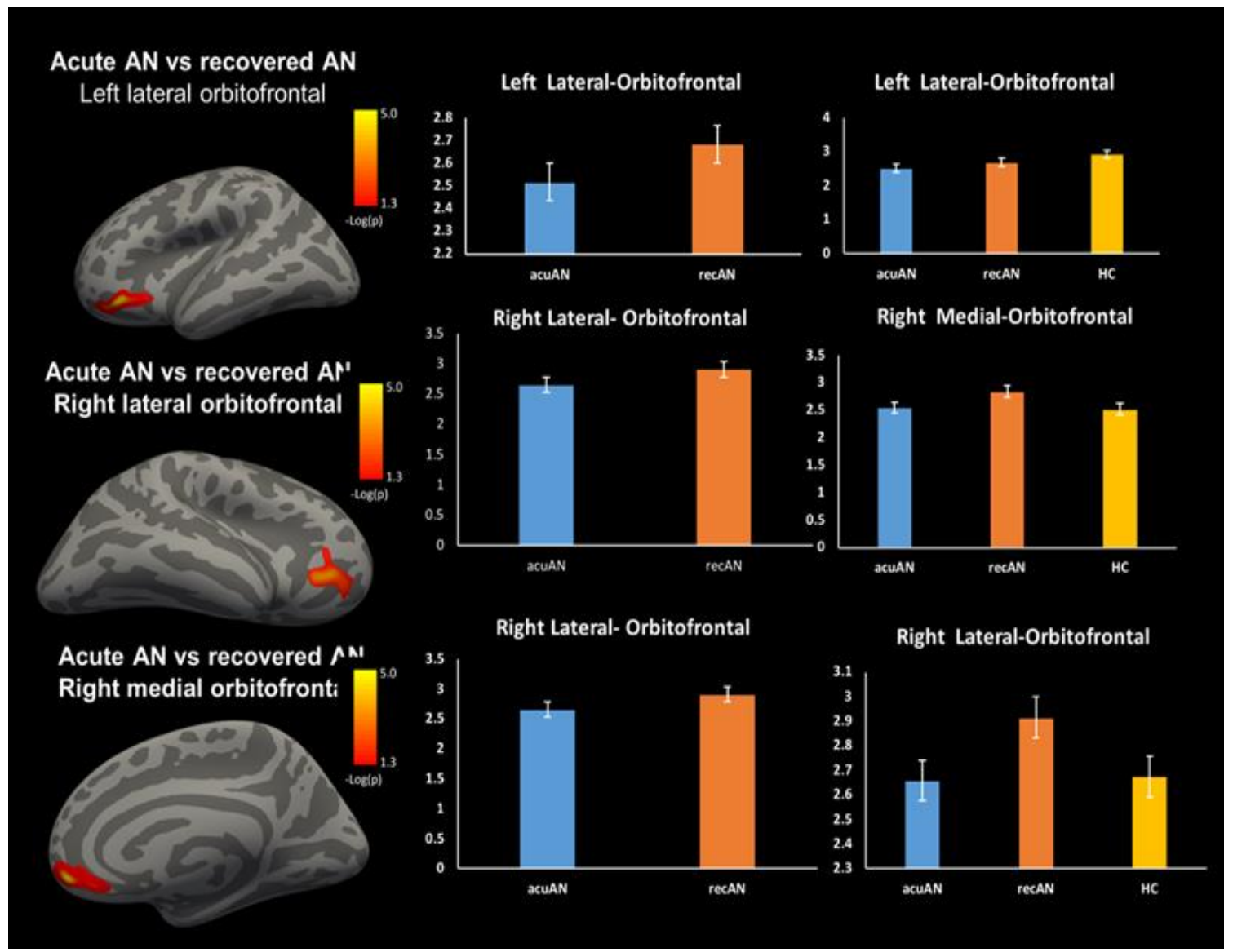

Figure 1. Differences in local CT between acuAN group and recAN group.

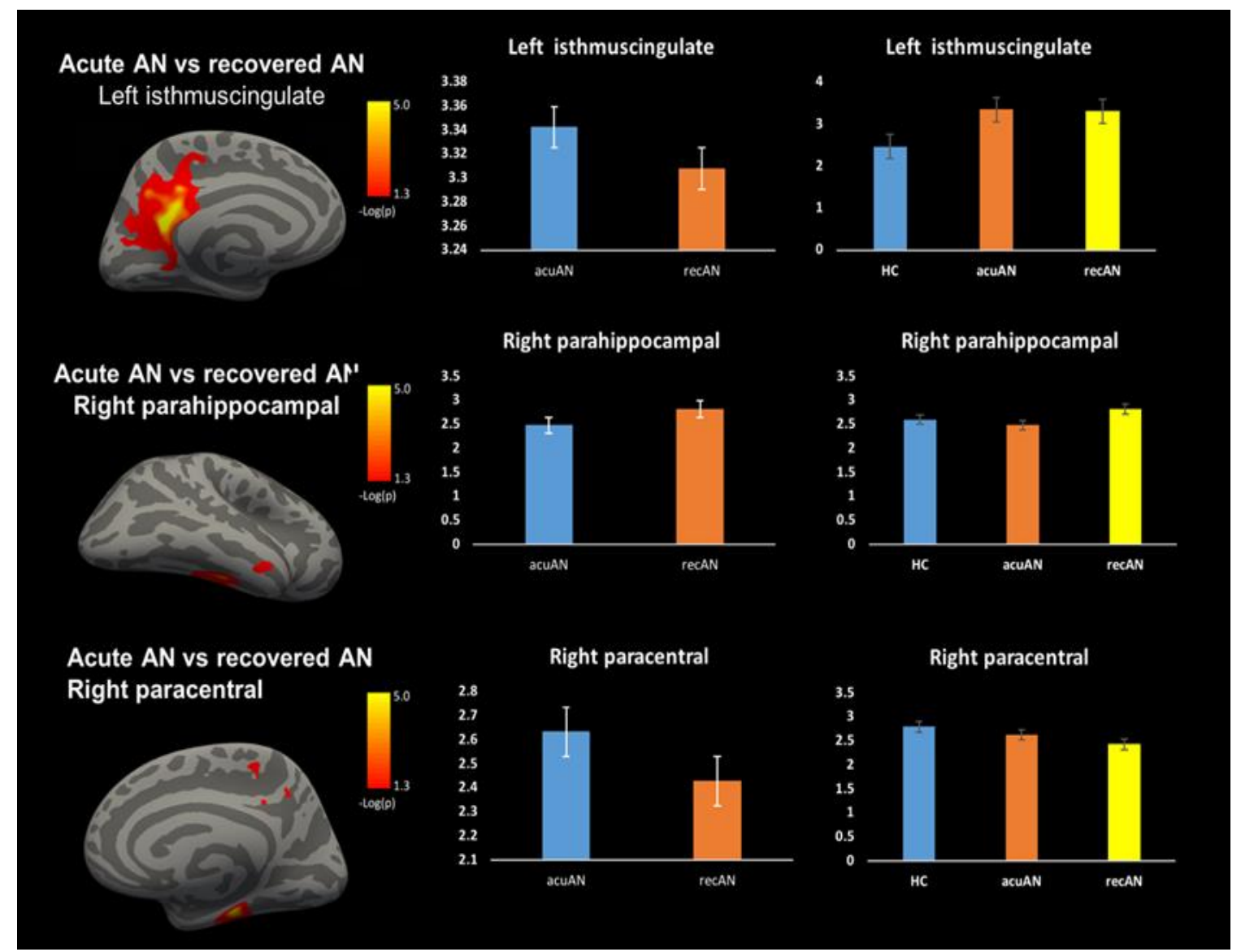

Figure 2. Differences in local GI between acuAN group and recAN group.

AN group showed greater values of GI in cingulate cortex and in paracentral lobule, lower values in right parahyppocampal region than recAN group and lower values of GI in left superior parietal cortex than HC. BN group showed lower GI in right frontal cortex compared to HC. BMI values correlated negatively with GI values in parahyppocampal and paracentral regions in AN and recAN groups.

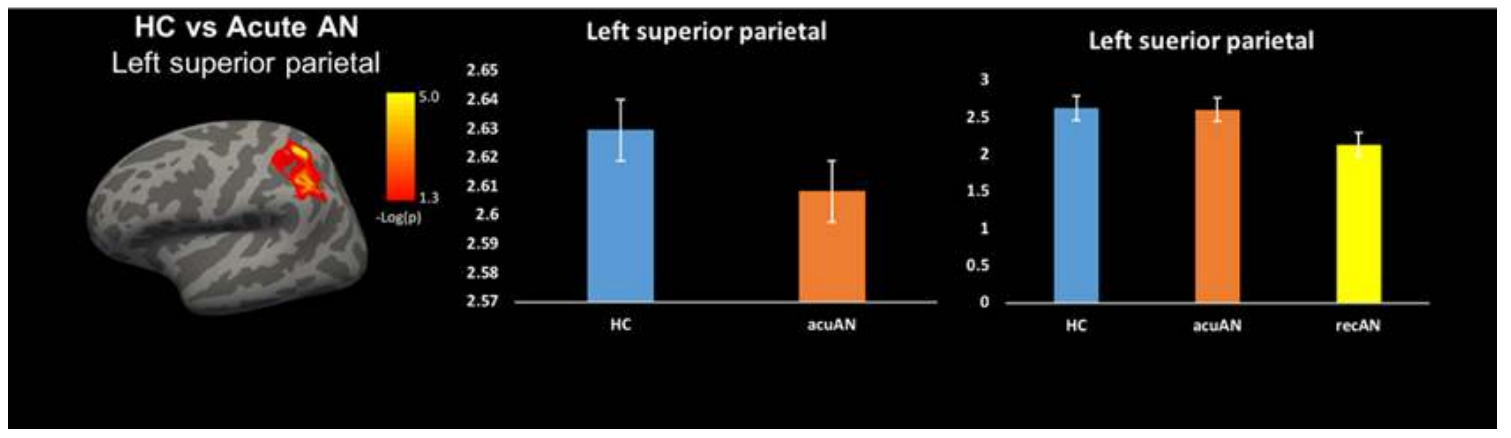

Figure 3. Differences in local GI between acuAN group and $\mathrm{HC}$.

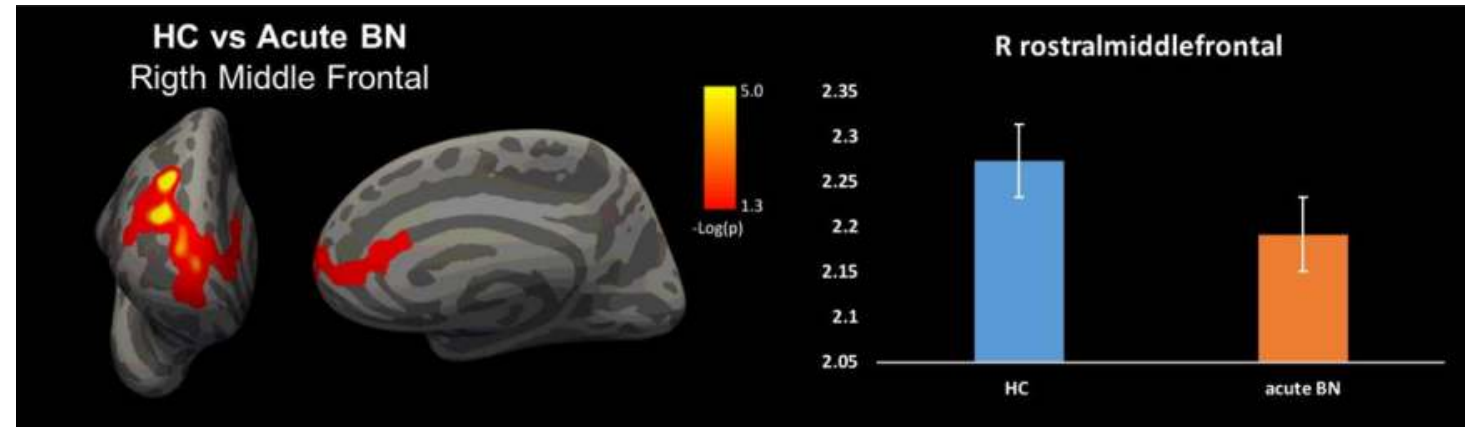

Figure 4. Differences in local GI between BN group and HC.

\section{CONCLUSIONS}

Present results provides evidence of CT and GI alterations in patients with AN and, for the first time, in those with BN. Although some of these differences were likely due to malnutrition, it is possible to assume that they may underlie psychopathological aspects of EDs, since the altered areas are involved in modulation of brain functions such as body image perception. 